\title{
Linked Information Visualization for Linked Open Government Data: A Visual Synthetics Approach to Governmental Data and Knowledge Collections
}

\author{
Florian Windhager, Eva Mayr, Günther Schreder, \\ Michael Smuc
}

Department for Knowledge and Communication Management, Danube University Krems, Dr.-Karl-Dorrek-Str. 30, A-3500 Krems, Austria, firstname.lastname@donau-uni.ac.at, +4327328932338

\begin{abstract}
Open government data initiatives provide citizens with access to the knowledge that governments have about their countries (such as data about people, resources, infrastructure, or services) upon which they act. Information visualizations can help to make sense of these complex data and knowledge collections, but are mostly used to shed light on subselections of data, without coordinated efforts to connect them to bigger pictures. In analogy to linked data initiatives, this article discusses methods and strategies to link visualizations in the government data realm and thereby to connect widely available local pictures and insights into more coherent global mental models. We expect related developments to provide benefits for communication professions like civic education and political journalism, and to enhance methods for cross-domain exploration and reasoning for linked open government data. Thus, linked visualizations aim for supporting students, readers, and citizens to meet a range of macro-cognitive challenges, which complex societies are facing in increasing amounts.
\end{abstract}

Keywords: government data, visual access, information visualization, linked data, linked images, cognition support, macrocognition, civic education, political journalism, linked open government data

\section{Introduction}

Democracies rely on the frequent participation of their populations in complex cybernetic circles of collective decision-making. Although these decisions (i.e., elections or referendums) can be based only on citizen's fuzzy feelings, sustainable self-government benefits from a deeper processing of political information and from rational reflections on the state of a collective environment ${ }^{1}$. 
Rephrased from a cognitive science perspective, reflections on external systems equal "mental models", which internally represent external constellations, and provide the basis for problem solving and acting (Johnson-Laird, 1980). Mental models can be more or less elaborated and coherent - based on the amount of information and its relations modelled - and thereby lead to more or less reflected decisions and actions. For political realities, mental models are constantly (re)shaped by public discourse (as decentralized multifrontal information processing), which again is co-created by professions like civic education and political journalism. Recently, the opening of governmental knowledge collections (Open Government Data, OGD) aims to grant insights into the black box of political systems and governmental activities (Ubaldi, 2013), which could ensure citizens' well-informed participation later on (Fig.1).

Figure 1: Mediating Institutions Enabling the Observation of Government Activities: Political Education, Political Journalism, and Open Government Data Platforms.

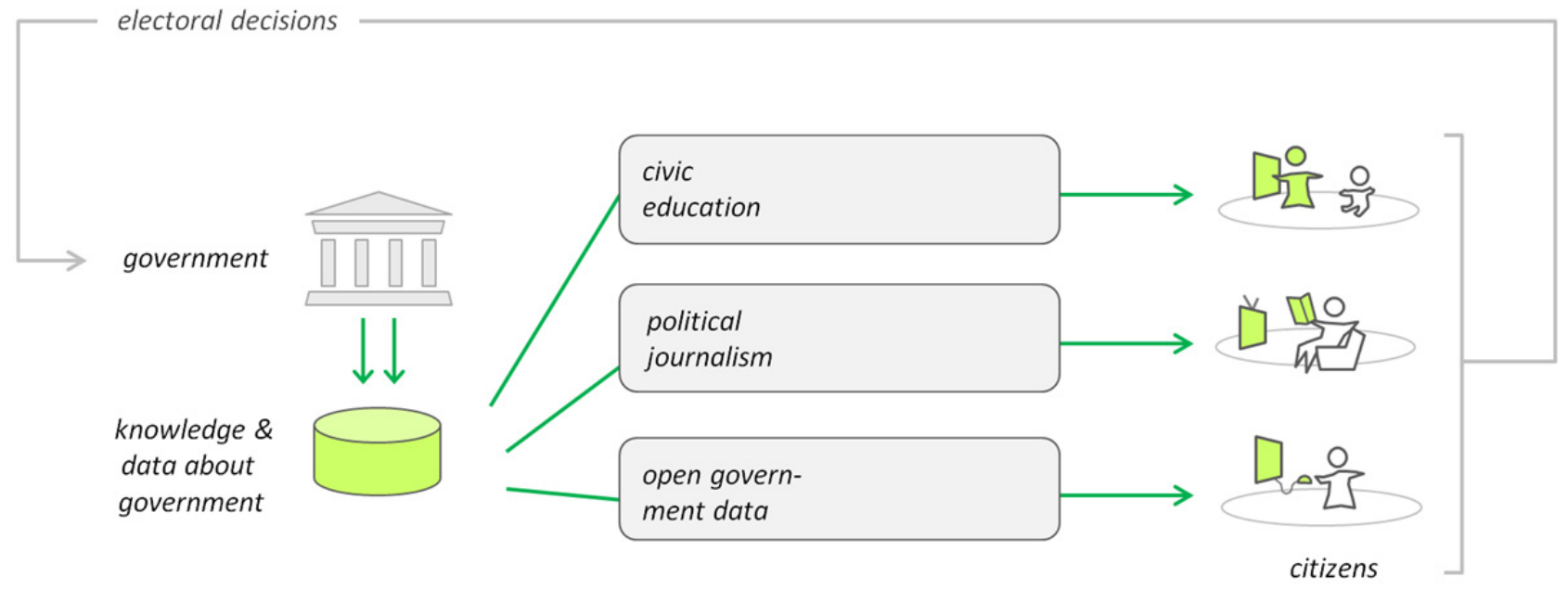

Across these institutions, mediating between government (data) and citizens, information visualization (InfoVis) plays an important role as a second modality, complementing the use of language. As diagrams in textbooks, as (interactive) graphics in data journalism, or as visual interfaces to OGD collections they convey visual insights into the structures and dynamics of political systems (Windhager \& Smuc, 2014) and support the construction of mental models of these systems. While these visual representations provide efficient overviews on a multitude of particular aspects of political systems, there has been little progress to interconnect these diagrams into bigger pictures on a visual basis. In this paper, we discuss how InfoVis interfaces can contribute to a better-connected understanding of political systems and government data by linking multiple visualization methods and perspectives. Similarly, to linked data initiatives, we

1 Rephrased by traditional terms of political science: On the state of a city (gr. polis) or country, and on a sound understanding of existing government structures (polity), their actors and activities (politics), and their various problem solving proposals (policies). 
argue that the linking of visual representations enables better sense making across usually unconnected data and knowledge silos, and that it can foster a more coherent and integrated understanding of complex constellations (including reflections on one's own position in the larger context).

From a cognitive perspective, linked information visualizations not only grant local cognition support on selected data sets with well-delimited complexity, but also aim for the support of meso- and macro-cognitive reasoning operations (Klein \& Hoffman, 2008) across a diversity of usually separated data domains, data dimensions, and views. Applied to civic education, political journalism, and open government data, linking visualizations raises the interoperability of existing diagrams - and therefore extends visual communication into more complex data and topic realms. We expect these considerations to provide a contribution to visual information integration, and thus provide techniques to support macro-cognitive reasoning and communicating (e.g., teaching) about complex systems.

To discuss possible approaches to these aims, the following sections will look the current state of government data, section 1, and InfoVis methods, section 2. Section 3 presents generic methods how to interlink InfoVis methods to generate bigger pictures of governmental constellations. We draw together an outline of a meta-representational scaffold for government data to enable complex visual reasoning in section 4 . Finally, in section 5 we reflect upon future implementation scenarios.

\section{Government Knowledge and Data}

What are the main components or elements that make up the specific configurations of complex self-governing systems (from administrative districts to contemporary nation states)? We outline some of the most common elements by a working ontology to better illustrate what "government data" usually is about. We consider this step - to make the provenance of abstract data explicit - as a relevant technique to specifically support the sense making of non-expert users of future interfaces - which helps to increase required levels of visual literacy of citizens by onboard means. ${ }^{2}$

2 We consider the presentation of abstract diagrams "ex nihilo", which could not be traced back to "real world"-entities, as one of the major (cognitive) obstacles to comprehend and understand the meaning of InfoVis interfaces (cf. the discussions of data provenance in visualization, Ragan et al., 2016). As such, we introduce a simple ontology illustrated by pictograms ("visual elementals") for showing the most relevant origins of government raw data first and cover the "who" or "what"-questions of fata lineage (cf. Ram \& Liu, 2009). These pictograms could be used as simple model kit to illustrate any selected constellation - and to trace its transformation into diagrams and visualizations (in civic education, journalism and OGD portals) later. Even though ontologies are arbitrary - and can always be done different (cf. similarities to they are indispensable to form sets and categories of highly diverse entities, which help to translate from the material world into visual abstractions later. 


\subsection{Basic Elements of Self-Governing Systems}

By the means of a simple ontology (Figure 2), we distinguish basic elements of political units (from here on: self-governing systems), which will reveal themselves to be the major data sources of any political scenario or constellation later. ${ }^{3}$

Figure 2: A Simple Visual Ontology of Major Elements and Relations in Self-Governing Systems, which act as major "data sources" in quantitative and visual approaches to political systems.

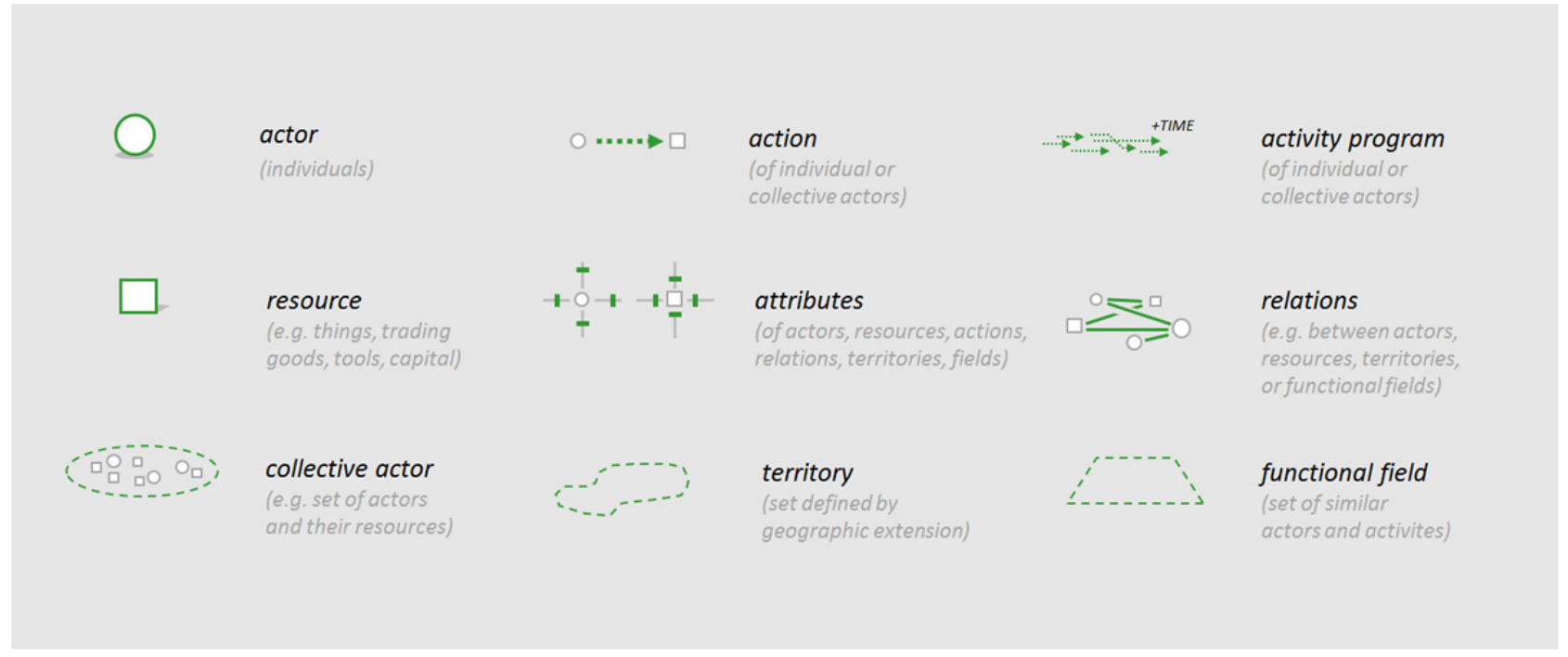

According to this ontology, self-governing systems manage interactions (depicted as links) between individual actors (depicted as circular nodes) and their relations (depicted as links) to resources (rectangular squares). These basic elements (actors and resources) are commonly grouping themselves by relational patterns (later: trees and networks) into various constellations of collective actors (delimited as dashed set-diagrams). These elements (actors, resources, links) can have multiple attributes (depicted as values on scales). All actors pursue their (individual or collective) goals by actions (depicted as arrows) and activity programs (sequences of arrows) over time. ${ }^{4}$ All resources, actors and their behavior are situated in material environments or territories (depicted as dashed shapes in geographic space) - and simultaneously affiliated to activity environments or functional fields (depicted as dashed shapes in social-topological space).

3 In contrast to existing general provenance ontologies like PROV (https://www.w3.org/TR/2013/RECprov-o-20130430/), we offer a visual ontology specified for self-governing systems. Though its elements can be largely mapped to the PROV ontology, some of them go beyond, e.g., by defining organized actions (PROV: activities) over time as activity programs (actions serving the same goals).

4 As the most basic activity program temporal continuation or self-reproduction (i.e., material and cultural reproduction) of actors could be assumed, complemented by varying programs to raise, maintain or lower specific attributes over time. 
This set of simple elements allows the reconstruction or simulation of any politically organized collective amongst others. Given any such setup, individual and collective actors are used to behave in dynamically shifting coalitions in relation to (mostly restricted) resources, which initiates adversarial (competing) or collaborating interaction patterns. Specific action patterns depend on a collective's complex coordination and activity program of how to achieve material and cultural reproduction as well as how to mediate individuals', the subgroups' or the collective's rationalities.

\subsection{Data Collection as (Self-)Perception of Self-Governing Systems}

Given any self-delimiting collective (e.g., a tribe, a city, a nation state, or supranational organization), governing agencies act as managing actors or organizations, guided by their specific management programs. According to dynamically and culturally changing customs, common requirements for these programs include the protection against external threats, the management of internal conflicts, general problem solving, promotion of collective welfare, etc. (Finer, 1997). ${ }^{5}$ To pursue these aims, governmental actors must observe the state of their collective and the state of their environment, which is achieved by distributed monitoring and corresponding data collection.

Linking back to the ontology of Figure 2, governments thus collect and process data on a wide range of their collective's elements (actors, relations, attributes, locations, etc.) to manage domestic affairs. This collection is usually complemented by a government's self-observation, like descriptions of its own structures and aims, and an ongoing monitoring of its own performance. Furthermore, based on reliable data, this domestic system data is commonly complemented by observations of other collectives to manage foreign or inter-collective relations, either conflicts or collaborations.

In open societies, these core collections of factual "government data" are complemented by a vital periphery of data and knowledge generated from external, non-governmental actors. This includes descriptions and data originating from journalism and media, from educational or academic organizations, open knowledge providers, as well as other NGOs (e.g., citizens' initiatives, opposition parties, think tanks). ${ }^{6}$ Even if we do not expect this complementary "NGO-

5 These traditional programs of "good government" obviously can turn into corrupted or deviant programs - including mismanagement, self-maintenance, oppression, exploitation, and rejection of legal accountability, etc., against which open information and transparency is seen as one of the most relevant strategies.

6 With this conceptual extension, perhaps one of the most interesting and challenging aspects of the political data domain comes into play, which is the polyperspectivic and therefore ambiguous nature of many data and descriptions. As descriptions of political systems can vary according to multiple possible theories, methods, perspectives, ideologies and values, and vary from affirmative to critical stances, etc., many forms of observer-related "uncertainty" or (multi)perspectivity must be also managed on the data level. To not generate unreflected propaganda on the (visual) analytics level, but to make conflicts of interests, perspectives, and power on descriptive levels transparent, we consider systematic 
knowledge" to be joined and integrated in comprehensive data collections anytime soon, we consider "linked data"-initiatives (see section 1.5) to provide the basic technological means to draw such different institutional sources and data silos together. As such we think it is essential to take future scenarios of information integration into account when it comes to reassembling the Political (Harman, 2014) from a linked information visualization perspective.

\subsection{Basic Data Types in Government Data Collections}

From an information technology perspective, government data collections as outlined above comprise all possible data types between structured and unstructured data. "Structured data" is commonly defined as machine-readable and (statistically) computable data with a high degree of organization, like data residing in a row-column-table or in a relational database. This type of data is often set apart from "unstructured data" like text documents, images or other multimedia documents, which obviously are "unstructured" only from the perspective of traditional parsing and decoding capabilities of early computers. ${ }^{7}$ As new techniques (like natural language processing, image and video analysis methods) find their way into governmental data processing too, it is safe to assume that shares of "unstructured" - and therefore "machine unreadable" data are constantly shrinking, while continuously extending the shares of knowledge that could be technically translated into structured data for further analysis and interpretation (Baeza-Yates \& Ribeiro-Neto, 2010).

Figure 3 offers a breakdown for a more specific distinction of prominent data types in government data collections, which has been adapted for structured data from Shneiderman (1996). While all these data types (1D, 2D, 3D, mD, tree and network data) could be found in government data repositories, the probably most frequent types are multidimensional datasets, listing various attributes for relevant entities (e.g., citizens, organizations, resources, environments), and geospatial data (e.g., on buildings, places, services).

\subsection{Opening Government Data Collections}

Government data collections have traditionally been handled as non-public, protected knowledge repositories, to provide operative knowledge for the use of administrative agencies only. While a significant amount of government data will always keep this restricted status, a

investigations into related data controversies as a highly relevant thread of development. At least it seems beneficial to base government transparency initiatives like OGD approaches on deepened data and design transparency standards (cf. Dörk, Feng, Collins, \& Carpendale, 2013).

7 Estimates about the amounts of structured versus unstructured data in organizations assume up to 80 or 90 percent of data being unstructured (Gantz, \& Reinsel, 2011). This number outlines the rather modest degree of overall data and information integration, which could be achieved by collections of structured (open) government data only (see section 1.4) - while simultaneously illustrating the relevance of further linking data technologies (see section 1.4) - or of information extraction technologies, bringing formerly unstructured data into the machine-readable (neo-structured) data processing realm. 
recent development has been the opening of non-sensitive parts to the public (Chun, Shulman, Sandoval, \& Hovy, 2010; Ubaldi, 2013).

Figure 3: Typology of Prominent Data Types for Various Subsets of System Elements

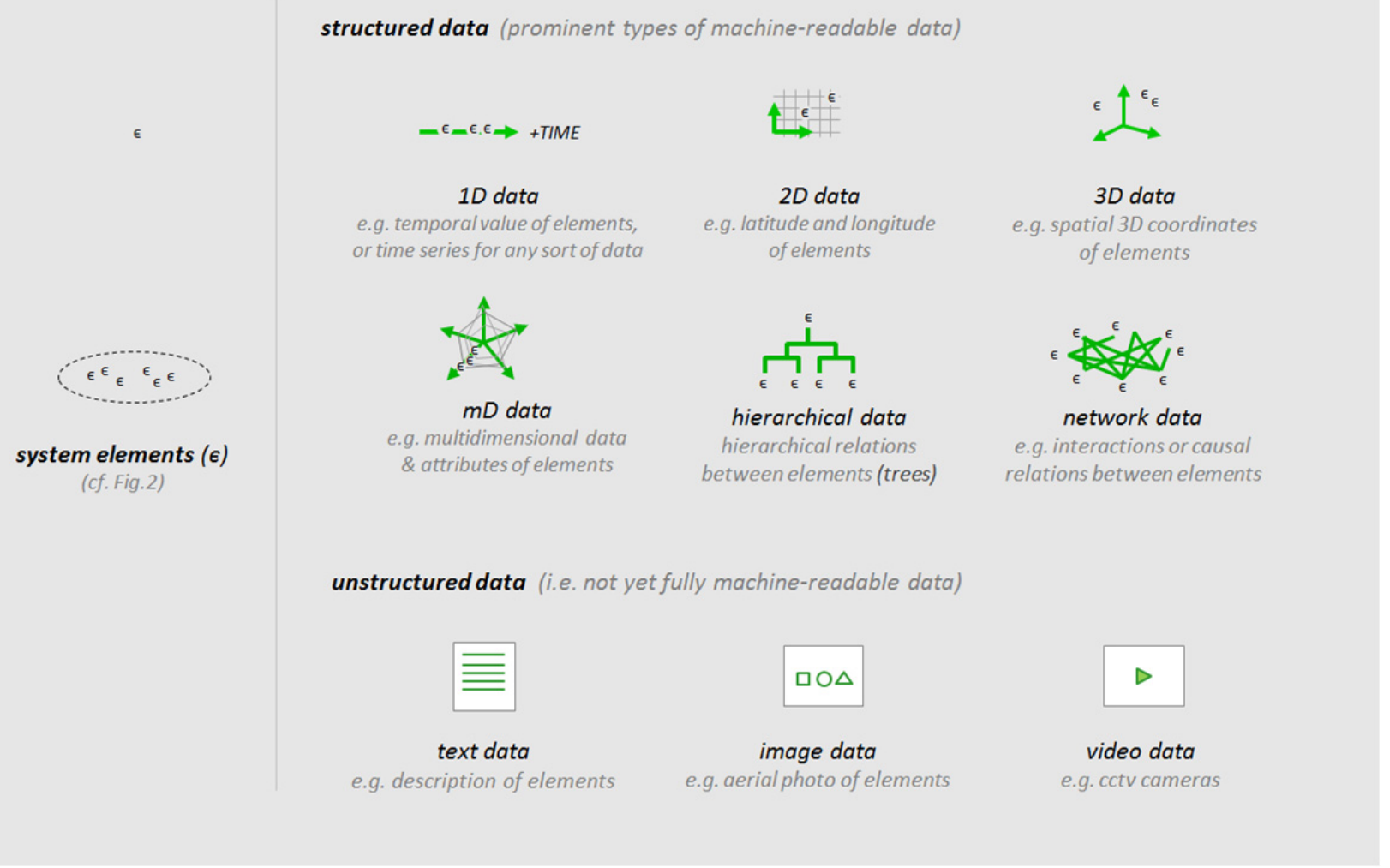

Open government data (OGD) initiatives strive for such a transition (amongst other communication measures built on new media technologies) to raise an administration's transparency and to foster public innovation, e.g., by enabling bottom-up approaches to application development. As indicated by Figure 1, these initiatives also foster general knowledge transfer, by granting citizens direct access to information about the state of their collective environment.

From an IT perspective, OGD platforms predominantly accumulate structured data for a large subselection of all system entities shown in Figure 2. Even though restrictions for access on entities (e.g., sensible data on individual actors, sensitive resources and organizations etc.) to prevent abuse exist, a wide range of general data becomes available for open access and analysis. For further analytical procedures and sense making, we will highlight the role of a whole range of information visualization methods (see section 2), which occasionally are already provided 
directly on OGD sites. ${ }^{8}$ While such accumulations of formerly closed and unconnected datasets and their opening via world wide web is of great relevance for government data integration and accessibility, OGD collections feature several challenges. Janssen, Charalabidis, \& Zuiderwijk (2012) exemplarily discuss institutional, task-related, user-related, legislative, qualitative, and technical barriers or challenges for the adoption of open data.

Two additional challenges deserve closer attention in context of our consideration: As with data provided by printed sources, OGD files commonly enable specific insights in various highly specific topics, but these insights and topics are often hard to contextualize, and do not necessarily add up to a bigger picture. This relates to both a certain lack of information architecture of OGD portals, and the restricted quantity of data sets, which even bigger portals could provide. Concerning content organization and information retrieval, OGD portals usually only provide several content categories, which are often aligned with the functional fields of government activity (like employment, health, education, or art and culture). Therefore, most landing pages only offer a lineup of these categories, embellished by pictograms (Figure 4), and complemented by a search functionality for metadata descriptions. After drilling down into these categories, no further information architecture helps to cognitively organize the complex multitude of diverse datasets, or to reason and navigate across category borders. On the other hand, the limited character of OGD portals results from them being subselections of structured government data only, while relevant other data and knowledge is distributed across a variety of government agencies websites - or stored in NGO data collections, like newspaper and education archives, open knowledge bases etc. Zooming out, this makes OGD collections just one archipelago in a scattered knowledge landscape of distributed sites, sources, and data silos. Aiming to transcend these unconnected topologies, linked data-initiatives bring highly relevant methods and techniques to the scene.

Figure 4: Data Categories on the Landing Page of the U.S. OGD Portal www.data.gov.

\section{BROWSE TOPICS}
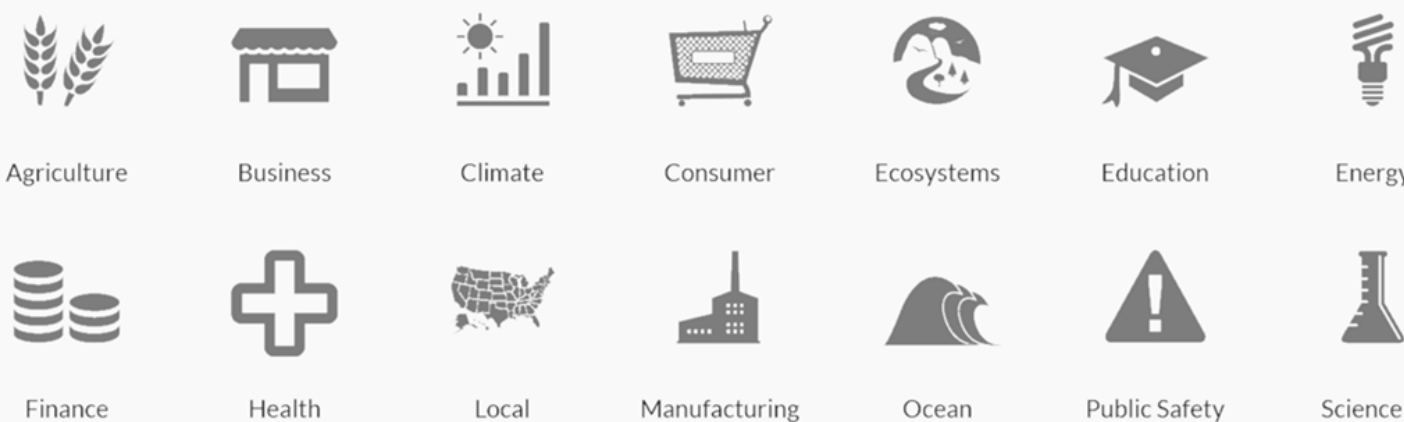

Business

Ecosystems

Education

Energy
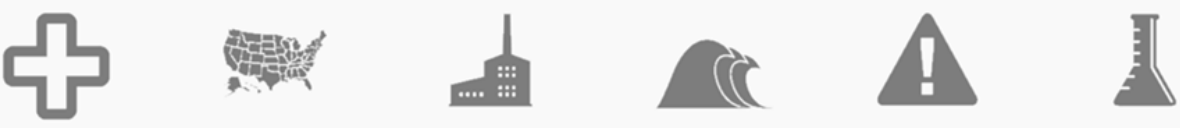

Health

Local

Manufacturing

Ocean

Public Safety

Science \&

Research

8 Further visual-analytical access is sometimes directly supported on site by the means of interactive statistical charts or maps (e.g. https://data.gov.uk). 


\subsection{Linked Open Government Data (LOGD)}

The concept of "linked data" originated from initiatives to further develop the world-wide web from a web of documents into a "web of data" (Bizer, Heath, \& Berners-Lee, 2009). Whereas the internet as a web of openly accessible documents had already transformed the representation and (re)organization of knowledge in an unprecedented way, its early-stage information architecture shows notable limitations with regard to (semantic) data granularity and interconnectedness. As a vast collection of mostly unstructured text documents and closed data silos, interconnectivity is provided by hyperlinks between whole documents only. By contrast, a web of data would resolve documents into more fine-grained constituents (i.e., its elements, cf. Fig.2) - and interweave these constituents within - and between - documents or data files by semantically meaningful (i.e., better specified) links. For this purpose, unique identifiers (URIs) are assigned to any relevant system entity, and a fine-grained ontology language (e.g., the resource-description framework $(\mathrm{RDF})$ ) provides a universal, machine-readable meta-language to make explicit how entities are specified by attributes and connected by interrelations (Klyne \& Carroll, 2006). Simply put, linked data technology transform collections of (formerly) unstructured and redundant documents into structured and interconnected data collections (knowledge graphs), which offers enhanced ways of searching, contextualizing, (re)combining and reasoning for machines or algorithmic actors (e.g., search engines, applications) - across formerly unconnected data repositories.

Figure 5: Linked Open Data Cloud Diagram, with Interwoven Government Databases and Sources (outlined in green) (Schmachtenberg, Bizer, Jentzsch, \& Cyganiak, 2014).

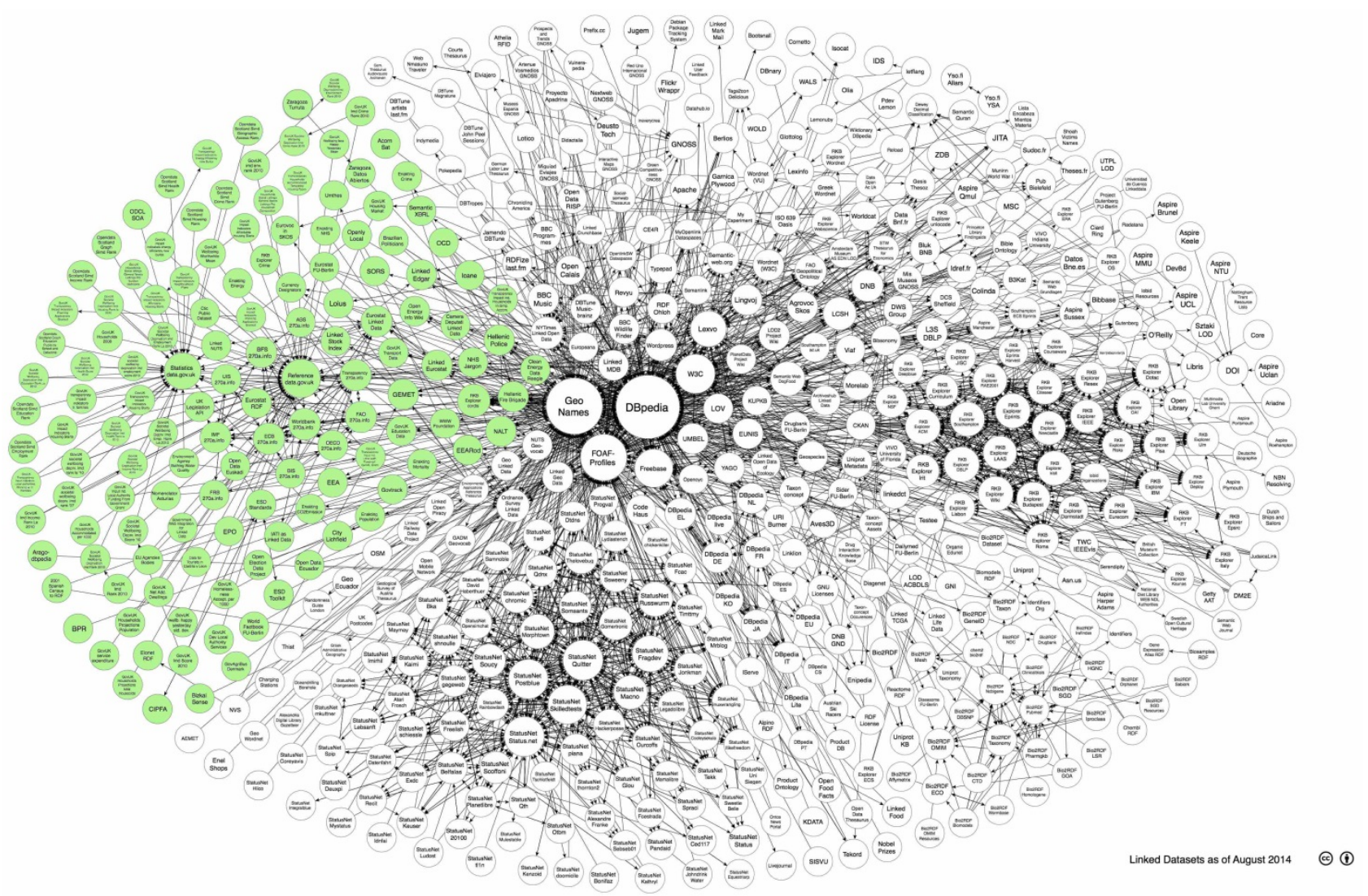


As a global and web-wide initiative, the linked data movement also started to interconnect and integrate government data (i.e., OGD collections) into a growing "global knowledge graph", thus upgrading them to LOGD (linked open government data) collections on the way (Ding et al., 2010). By these developments, the aforementioned limitations of OGD collections (cf. 1.4) become smoothed out: i) the limited amount of data grows by linking formerly separated governmental data silos with other-governmental and non-governmental data; and ii) a former lack of internal data organization, information architecture or cross-document and cross-category integration becomes replaced by rich relational structure between documents, transforming separated and intransparent texts or data files into structured local RDF graphs, and linking these local graphs into a global interoperable graph (Fig. 5).

Thus, this large-scale integration brings along new options for accessing and querying government data and knowledge (Alani et al., 2007). Technically, a wide range of participation options arise - from linked searching and browsing, to linked reasoning about linked data constellations and their context, and eventually linked acting (e.g., as citizens' initiative) - as long as citizens can also generate linked meaning (read, process and interpret), turning data into (linked) mental models and these mental models into actions.

Yet practically, the increasing number of resources and their data and metadata heterogeneity also pose significant challenges, which must be answered with complex querying or data mining methods. Dadzie and Pietriega (2017) state that these solutions often require high technical knowledge to query databases and automatically build barriers for adoption and use of these tools by citizens. To meet their needs, easily accessible, user-friendly Information visualizations could be a solution. In the following, we will not address the data mining and manipulations techniques necessary to link open data and assure their quality - even though we are aware that these techniques pose numerous challenges on their own'. Instead our focus is upon the question how successfully linked constellations of OGD can be presented visually, to support citizens without technical knowledge in building a mental model of these data. Methods of information visualization already deliver a powerful set of tools to do so - for relatively simple datasets. The following chapter will line up some of the most prominent methods of visual data analysis for local and well-delimited data selections, while possible techniques for linking separated visualizations into bigger pictures government data will be investigated later.

9 For reflections on the comprehensive challenges connected to the upgrading of data collections to LOGD collections (amongst other methods and efforts requiring data mining, link discovery, and record linkage techniques) see Ngomo \& Auer (2001) or Villazón-Terrazas, Vilches-Blázquez, Corcho, \& Gómez-Pérez (2011). 


\section{Information Visualization as Visual Access to Government Data}

Information visualization (InfoVis) amplifies and supports human reasoning with abstract data by providing interactive graphic representations (Card, Mackinlay, \& Shneiderman, 1999). Such representations use the spatial relations on a display congruently to the numerical or conceptual relations of abstract data, to exploit the highly-practiced skills of spatial human reasoning for abstract reasoning (Tversky, 1993). The resulting images help to build up visuo-spatial "mental models" of abstract data, on which further cognitive operations can take place (Liu \& Stasko, 2010). How abstract data is assigned to spatial positions, shapes, relations and further visual variables of a diagram or display is governed by a variety of InfoVis methods, which have been developed to support cognition with different data types (cf. Figure 3; Heer, Bostock, \& Ogievetsky, 2010).

The following sections outline some of the most prominent methods and illustrate their applicability for government data of well-specified, local complexity (cf. Windhager \& Smuc, 2014). Starting with sets (to visualize any subset of elements), the focus shifts to graphs (to visualize relations as trees, concept maps, or networks), to statistical charts (for visualizing attribute distributions), and to maps (to visualize geospatial data), to be complemented by reflections on how to visualize time-orientation for any sort of data and InfoVis method.

\subsection{Set Diagrams}

As a very basic information visualization technique, sets allow to graphically assemble any selection of elements into complex or compound units of analysis (Alsallakh et al., 2014). Regarding the ontology of elements (Fig.2) sets can exemplarily group actors, resources, and their related actions into collective actors (e.g., by dashed lines), which range from most diverse types of organizations to large-scale political or functional collectives. The outlines of political collectives then could be visualized due to various aspects: They could be visualized as they are arranging themselves in geographic space (i.e., as territories on maps, cf. 2.6), or by transforming the given physical arrangement into an alternative layout, which helps to expose other aspects of the outlined element collections, like inherent relational structures (cf. 2.2, 2.3, 2.4), attribute distributions (2.5), or dynamic behavior (2.7). "Information Visualization" as set of imaging methods thus is often set apart to methods of "Scientific Visualization", which keep existing arrangements or already given spatial layouts intact (like photography, cartography, or 3Dmodelling).

Figure 6 shows different options for the visual-analytical (re-)arrangement of a political collective (center) - with a physical or geographic projection on the left side, and a functional projection (dividing itself into societal fields) based on a simple set on the right. Whereas the left side allows to see geographic distribution, the right side makes functional distributions and division of labor visible. Functional fields are large sectors or actor networks dedicated to the provision of specific functions for collective reproduction, like the economy, science, media, law, education, the health system - or the political system itself (Stichweh, 2013). From a government data perspective, sets provide the basic means for visually distinguishing political units from their 
environment - and to go into further visual analysis by the means of other InfoVis methods as a next step, including methods to shed light on the internal organization of a unit's decision structures and power relations.

Figure 6: Various Set Diagram Layouts, showing the the geo-spatial projection on the left (cf. 2.6), and a functional set projection showing division of labor on the right (cf. 2.3).

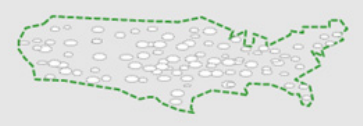

geo-spatial projection based on physical arrangement (cf. 2.6)

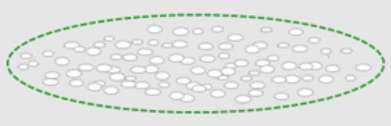

set of collective actors

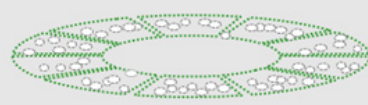

functional projection based on division of labor, cf. 2.3

\subsection{Tree Diagrams}

Trees are visual representations for hierarchically structured phenomena and their data. Governments as managing organizations with far-reaching executive functions provide a classic example for complex and widely ramified hierarchies. As a well-known effect, decisions from top positions (presidents, secretaries, officers, etc.) could be assiduously transmitted and multiplied via branching chains of command to be implemented as executive measures in a collective's lifeworld and environment.

Tree visualizations come in different versions - as node-link diagrams (also dendrograms, or directed acyclic graphs) or as treemaps or sunburst diagrams, using space-filling layouts (Kobsa, 2004). Figure 7 shows three simple examples for tree diagrams, schematically modelling the traditional forms of government already known to the ancient world (i.e., monarchy, aristocracy and democracy) as organizational charts. On some OGD portals, the whole complex structure of modern day governments is visually accessible by interactive organigrams (e.g., https:// data.gov.uk/organogram).

As such they help to understand, how an administration divides its labor across governmental departments (or ministries) that are responsible for the political administration of a society's public sectors or functional fields (see Figure 6, right). The concentration of power at the top of a tree also shows how this position is a structurally critical and neuralgic one, as it can be abused easily. Consequently, controlling mechanisms like electoral control (or temporal separation of powers) has been developed for democratic systems, which adds a decisive bottom-up directed feedback loop to the governmental hierarchy (Fig. 7, right) to periodically reelect or renew their executive actors. 
Figure 7: Tree Diagrams Displaying the Traditional Distinction of Forms of Government.

tree diagrams

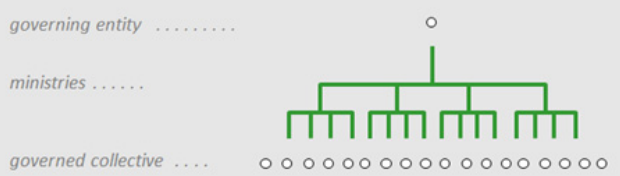

governed collective monarchy / tyranny

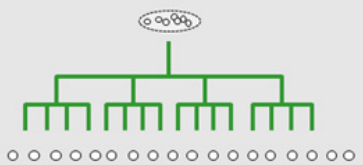

aristocracy/oligarchy

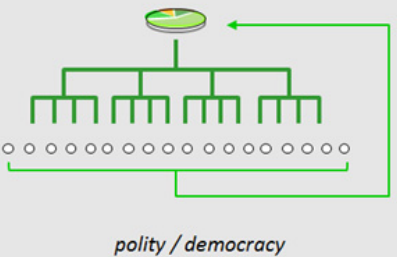

polity / democracy

\subsection{Concept Maps}

Concept maps are a subclass of node-link diagrams, which also help to understand a wide range of cybernetic government principles, where flows of power and control should be modeled. In such constellations, nodes are mostly collective actors or government institutions, and links are usually relations of command, advice, control, or legal supervision. Apart from that, nearly any other conceptual constellation could be modeled as concept map, where nodes can be all possible semantic entities, which are - according to a theory or concept - somehow interconnected or influencing each other. Often the layout of concept maps is generated manually, so that the readability of the whole constellation is maximized (Kitchin \& Freundschuh, 2000). Figure 8 illustrates two relevant concepts of self-governing systems as concept maps, amongst which are the separation of governmental powers (left) and a collective's division of labor (right).

Separation of powers: As they centralize executive power and legitimate use of force, the offices of governmental hierarchies face a constant risk of abuse. Whereas the promotion of collective well-being would be the traditional "good-government" program, the nepotistic misuse of powers and resources is the ever-present risk of political corruption, promoting the well-being of office holders or other ingroups only (Rose-Ackerman, 2013). Figure 8 (left) shows a schematic representation of an architecture of control, which keeps power systems checked and in balance and is commonly defined by a political constitution. Power thus is controlled by i) subordinating executive power under a body of laws, ii) establishing mutually controlling government branches (traditionally executive, jurisdictional, and legislative), and iii) frequently evaluating the performance of this system by democratic evaluation cycles (i.e., elections), as illustrated by Figure 7.

Division of Labor: Functional differentiation (or division of labor) can also be said to provide the reproductive basis for modern societies (Fig. 8, right) (Stichweh, 2013). Functionalist takes on social systems are used to model societies as ensembles of several functional subfields, with each field contributing essential functions and services to each other and the whole collective. Basically it is this network of large and complex fields (economy, science, media, education, health, culture, 
etc.) that has to be administrated and managed - at least where self-organizing (bottom-up) principles like a free market do not provide satisfying results. ${ }^{10}$ Government departments (or ministries) thus are the main governing subunits, which focus on the administration of these functional subfields. Naturally, it is also these fields, where the lion's share of open government data comes from - and which deliver the main data categories for OGD collections (Fig. 4).

Figure 8: Concept Maps of the U.S. Separation of Powers (left), and Large-Scale Network of Functional Fields, which are providing a collective's reproductive functions (right).

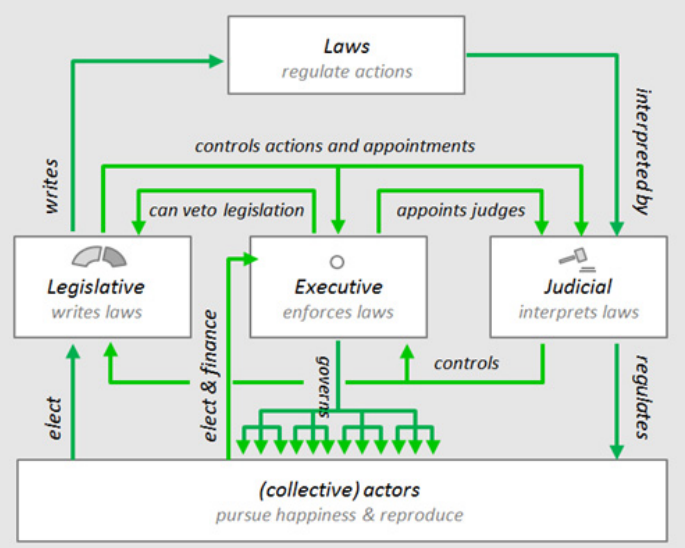

Concept: U.S. Separation of Powers

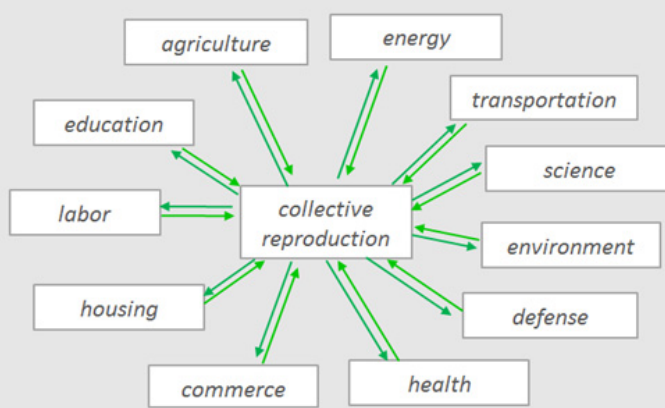

Concept: Collective Reproduction Network

\subsection{Networks}

As one of the most prominent layout methods for node-link diagrams, networks as force directed graphs can expose topological patterns within any constellation of relational government data. Possible elements to be modeled as nodes could be actors, resources, collective actors, or whole nation states, whereas links could be provided by most diverse sorts of relation and (inter)action patterns between the individual collaboration to the international relations level. If data on one or multiple types of relations is given (e.g., flows of trade, air traffic, or bilateral agreements between states), the layout of force-directed graphs is provided by spring embedder algorithms, which draw together nodes with strong relations and separate less connected nodes. The resulting graphs show topologies with clusters, wholes and proximity or distance of actors in social space.

10 It is well-documented, that the ratio between private or public (self-)management - i.e. to which extent and by which size a government should play a role in the activities of a collective - is amongst the most prominent and chronically controversial topics, which each collective has to frequently decide and reevaluate by itself anew. 
Figure 9 (left) illustrates this imaging procedure using international trade data (adapted from Krempel \& Plümper, 2003). On this macro-level, networks can help to visually analyze the multilayered networks of international relations between political collectives, where attracting collaborations and distancing controversies are shaping a map of the global political space. When it comes to the visualization of government data, we consider these topological maps to play an equally important role as geographic maps (section 2.6), even if they are still rarely seen in everyday articles or textbooks.

Figure 9: Force-Directed Network Graphs (left) and Possible Close-Up Layouts for Nodes Provided by Radar Charts (right), to represent multidimensional statistical data of nodes (i.e., countries).
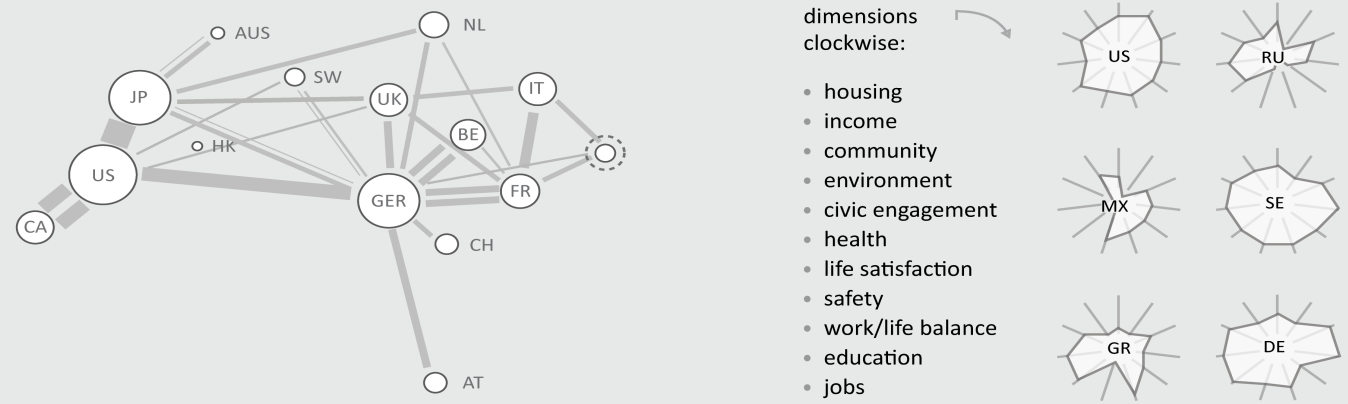

\subsection{Statistical Charts}

The most widely used InfoVis methods to visualize multidimensional data in the field of OGD portals, political journalism and education are statistical charts. Whenever one or multiple attributes of certain system elements (actors, resources, collective actors, opinions, etc.) are known, they could be transferred into a chart according to standardized layout principles. When addressed as a whole, statistical charts include dozens of different layout principles, but visual literacy for how to interpret e.g., bar, pie or line charts is high (e.g., Few, 2004). As a comprehensive toolkit, such graphs and charts help to visually analyze a wide range of quantitative data, gathered and accumulated by governments. Figure 9 (right) shows how to encode multidimensional attribute data of complex entities (i.e., countries or self-governing systems) into the length of radial axes of radar charts. More generically, figure 10 shows how multidimensional attribute data (left) could be transformed and encoded into any other visual 
variables (like $x$ - and y-position of marks, size, length, angle, color, etc.) of the whole panoply of statistical charts (cf. Schreder, Windhager, Smuc, \& Mayr, 2016).

Since democratic political systems process all their decisions (e.g., policy directions, options, scenarios, and the composition of their governing bodies) by the formation of majorities, it comes as no surprise that diagrams which show distributions (e.g., pie or ring charts) or provide precise quantitative comparisons (e.g., bar charts) are among the most widely used options when it comes to the coverage of public opinions and elections. Aside from these usual suspects, a range of advanced methods (e.g., parallel coordinates or radar charts, Fig. 10 right or Fig. 9, right) are often used to gain insights into characteristics of multidimensional data entities, and to support political decision-making.

Figure 10: Various Statistical Charts (right), Encoding Multiple Data Dimensions of Elements (left) into Visual Variables (e.g. position, length, angle) to enable visual analysis of data distributions.

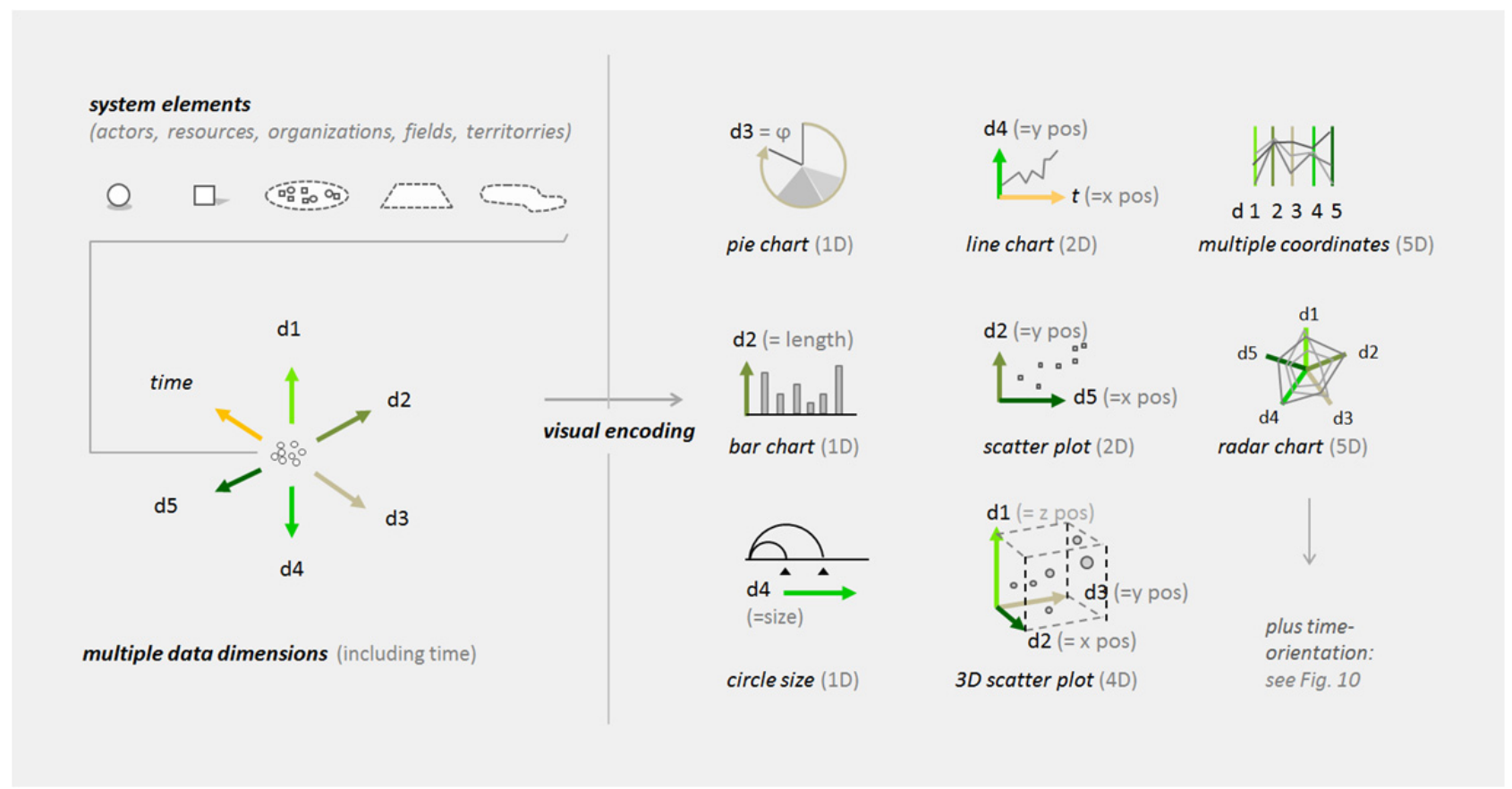

\subsection{Maps}

Geographic maps finally represent the locations and territories inhabited by collectives (cities, districts, countries) by redrawing their positions within physical borders and areas from a bird's eye view. As scaled-down representations of physical environments, they do not show abstract data in the beginning, but could be enriched and visually elaborated with colored layers (as choropleth maps) to encode values or attributes which would stay invisible to the naked eye otherwise (Figure 11, left).

Building on geographic maps, cartograms preserve some of their aspects (like relative positions of territories), but adapt or distort other aspects according to a salient visual variable, which helps to 
see the known world (or a selection thereof) under from a newly weighted perspective. Dorling cartograms (Dorling, 2011) exemplarily preserve the locations of political entities, but represent them as circular areas whose diameters depend on selected attributes of the inhabiting selfgoverning system (Figure 11, right).

Figure 11: A Political Map (left) and a Dorling Cartogram (right), with the former using color-coding to visualize the values of attributes of political units, whereas the cartogram uses diameter of (circular) territories to encode values.
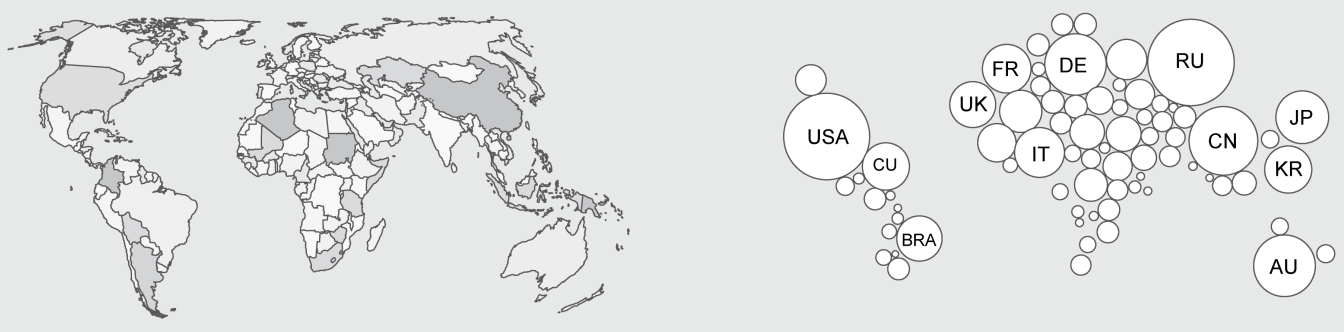

These circular representation of self-governing systems closes a morphological circle back to the first section of this section (2.1), where set diagrams also outlined social units as circles - and provides a toolkit of possible perspectives on government data, which show a strong potential to be instructively linked and combined into InfoVis interfaces of a higher order of visual and conceptual complexity further down.

\subsection{Visualization of Time-Oriented Data}

A challenge affecting all InfoVis methods seen so far is time-oriented data on any short or long term dynamics of the depicted constellations. Common solutions (see Figure 12) to visualize change over time on static data carriers like paper include juxtapositions of temporal snapshots (Fig. 12, from left) or superimposition of temporal layers with different line colors denoting different temporal states. On screens, animation can also be used to display temporal change, by altering an image over time. Likewise, 2.5D layouts can be used to stack temporal layers and show temporal trajectories in the interspace (Windhager, 2013).

Furthermore, the implementation of interaction methods is a standard way of coping with challenges of visual complexity. These methods help to reduce the overall information in an image by only showing specific data on demand. By making users part of the imaging procedure, the explication and exploration of a complex data set becomes user driven, and thus cognitive 
operations (like the comparison of shapes or different points in time) are visually supported on demand.

Figure 12: Methods to Visualize Time-Oriented Data (here with two time slices $t 1$ and $t 2$ ) include juxtaposition, superimposition, animation, and 2.5D layout, including interaction methods.
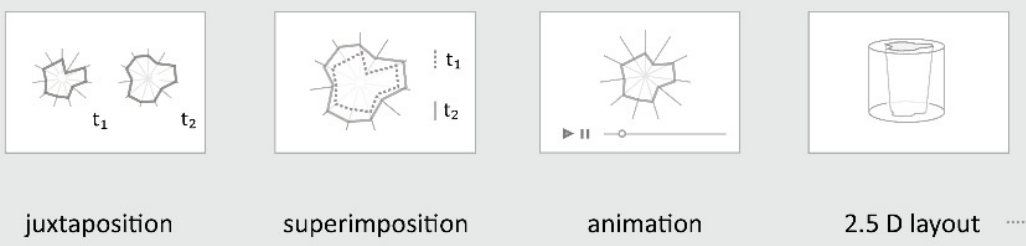

2.5 D layout

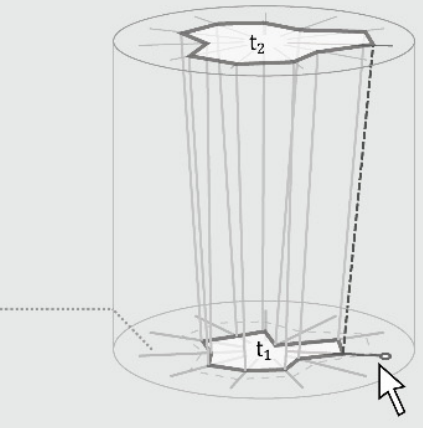

+ interaction

\section{Linking Information Visualizations}

The assembled presentation of prominent InfoVis methods already demonstrates how they can support citizens' cognition to make sense of abstract data collected by government and nongovernmental organizations. They can illustrate abstract concepts or disclose patterns in the numeric tables of data collections, which could be visually rearranged, explored and interactively analyzed. Some of these methods (like maps or statistical charts) are already firmly established in education and journalism - and are often also already implemented in OGD collections to visually analyze datasets without the need to switch to external tools (e.g., U.S. LOGD portal https://www.data.gov).

Yet, when it comes to a macro-cognitive syntheses and analytical tasks across separated data domains and constellations, these methods do not yet readily come together in a bigger picture. In analogy to language-based information processing, they show a well-defined local "visual syntax" (Engelhardt, 2006), which yet remains on the level of single sentences or paragraphs, whereas a visual macro-syntax (or "text-syntax") on how to create more complex representations out of these single views is missing. Speaking from a mental models-perspective, each InfoVis provides valuable local parts (submodels), but the creation of global mental models on a visuo-spatial basis remains a largely unresolved challenge (Schreder et al., 2016).

Even if the "linked open data" cloud - as a basis for visual analysis - already connects large numbers of datasets with a rich web of ontological and relational metadata and unique identifiers, the current state of research on "linked open data visualization" (cf. Pena et al., 2015) does not go 
beyond supporting local (micro)cognition, but rather focuses on how to automate the creation of local visualizations from any given local LOG dataset. ${ }^{11}$ We consider the result - i.e., a cognitively challenging multitude of heterogeneous and unlinked microvisualizations to be a systemic issue and grand challenge for future InfoVis interfaces (Windhager et al., 2015), and will tackle this challenge for LOGD by discussing methods to overcome these particularistic perspectives in the following sections.

\subsection{Linking Visual Representations via Hyperlinks}

The operating principle of hypertext - to connect textual information in any required non-linear fashion - has been transferred to the linking of pictorial representations and implemented in various systems and tools (e.g., ThingLink https://www.thinglink.com/, or HyperImage http://hyperimage.ws/de/). This technique enables users to link images (as well as any other information modalities like texts, audio, etc.) by specifying picture details as hyperlinks that could be activated by proceeding to a new image showing something related (e.g., a close-up, another perspective, another layout, etc.).

As a general linking technique, this method enables the creation of any intended pictorial network, which could be "surfed" in a sequential fashion, so that single views add up their content to a more complex constellation. As a limitation or cognitive challenge, the connection between visual representations is provided or realized by a "jump", i.e., by a disruptive move, where the kind of linkage - or the meaning of the transition - is not elaborated. With the progression from one image to another unrolling over time, the meaning of the transitions-as-links could be obvious, but is not guaranteed to be so in many cases of complex data interrelations. In such cases, more explicit and relationally rich linking techniques must be developed. ${ }^{12}$ Furthermore, sequential linking techniques are known to rely on (and soon overstrain) attention and working memory, when it comes to internally representing the whole picture. In contrast, methods of parallel views keep the content accessible and allow for repeated returns of focus of attention, as well as a zoomout to generate overview representations and mental macromodels on demand.

11 A recent review on LOD visualization by Dadzie and Pietriga, (2017) showed that only 5 out of 17 visualization tools used some kind of coordinated views, but did not include other types of linking visualizations. In their review with focus on big linked data, Bikakis \& Sellis (2016) stress the increase of challenges by large and time-oriented data volumes, but also emphasize how most of the visualization systems show high performance with scaled down datasets only, and do not look into multi-method integration as a strategy to cope with data and subject complexity.

12 On a related note: If some approaches to complex mental models do not engage in such sequential hyperlinking, but - in a parallel analogy - just visualized unspecified linked content or concepts as network graphs, then the questionable method of modeling mental models as semantic hairballs is the result. While both methods (i.e., hyperlinking content and organizing content as network graphs) could foster the understanding of small linked datasets, increasing temporal or parallel visual complexity will make cognitive systems meet their integration limits soon. 
Figure 13: Linking techniques for Multiple Visualizations include hyperlinking (top left), narrative integration (top right), integration by coordinated multiple views (bottom left), and integration by visuospatial macro-architectures (bottom right).

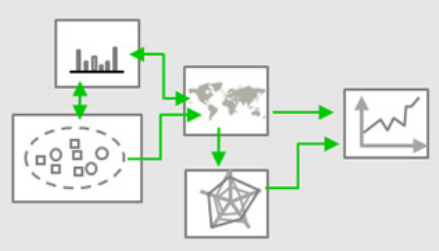

1) Linking Multiple InfoVis by Hyperlinks

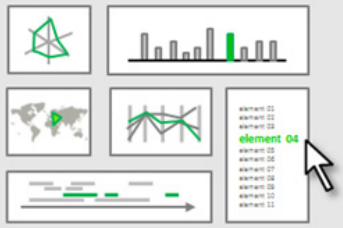

3) Linking InfoVis as Multiple Coordinated Views

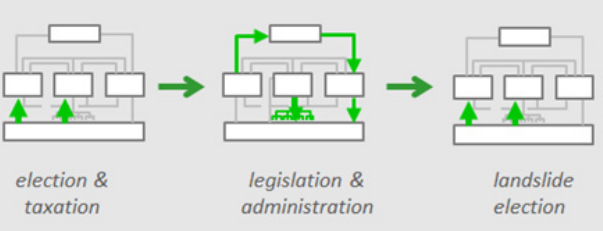

2) Linking InfoVis by Narrative Guidance

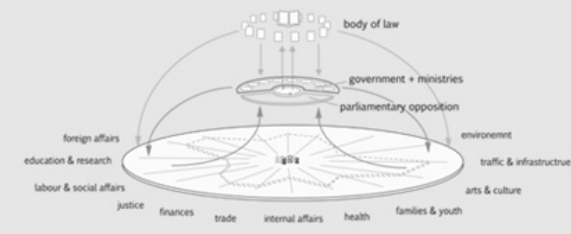

4) Linking InfoVis by a Spatial Meta-Architecture

\subsection{Linking Visual Representations via Narration}

Narration is most probably the longest-serving linking method for pieces and parts of information when it comes to language-based communication. Using narrative linking techniques in information visualization is a rather recent trend (Kosara \& Mackinlay, 2013). On a picture-basis it either manifests as a narrative walkthrough through a sequence of images, establishing interconnections by verbal or textual transitions, or as transference of narrative techniques into the visual design space (Segel \& Heer, 2010). Examples for techniques that draw upon narrative principles include not only film or animated visualizations, but also comics, slide shows or flow charts.

Whereas exploration-driven approaches (including hyperlinked images) to InfoVis do not prescribe any specific or linear order for engaging with representations, but allow for free investigation, a narrative (author-driven) structure guides the reader through the material. In between a balancing of author- and exploration-driven InfoVis is also possible, e.g., by drill-down structures, which visualize a general theme and allow users to choose between different subtopics, which again are heavily authored (Segel \& Heer, 2010). On an abstract level, visual storytelling allows recipients to construct mental submodels first (e.g., models for analytical perspectives), and support their global connection by providing sequential links between these parts. Also with this 
technique, working memory should keep the submodels present over time, and achieve their interconnection to a macromodel in retrospect (Schreder et al., 2016).

\subsection{Linking Visual Representations by Coordinated Views}

As another well-established linking technique, multiple coordinated views offer multiple perspectives on complex data sets in parallel (Roberts, 2007). Thus, multiple coordinated views are usually accessed in a side by side (i.e., juxtaposed or other composite) arrangement. With such an arrangement, observers can construct mental submodels for each view, and learn more about their interconnection by bringing coordinated interaction techniques into play.

The most common of these linking techniques are coordinated highlighting, or linking and brushing, but also synchronized panning, scrolling or zooming. These interaction methods provide instant visual modifications (as temporally synchronous changes) for the same data elements in different local views, which facilitates their perceptual integration. As a limitation, the spatial separation of multiple views requires perception to focus on multiple areas sequentially, thus necessitating split attention effects (Ayres \& Sweller, 2005). Furthermore, multiple coordinated views are commonly arranged side by side, without making their arrangement principles explicit, or without encoding meaning into their mutual positions - or into the white space in between. In contrast, visuo-spatial macro-architectures (see the next section) provide such an overall layout for conceptual orientation and multi-perspective integration.

\subsection{Linking Visual Representations by a Spatial Macro-Architectures}

With the term macro-architecture, we refer to any visuo-spatial meta-layout that supports the creation of a mental macromodel similar to an overview function (cf. Shneiderman, 1996). While it does not have to (e.g., Valsecchi, Abrate, Bacciu, Tesconi, \& Marchetti, 2015), such an architecture can also make use of three-dimensional space to present an information architecture that also instrumentalizes an additional data dimension regarding 2D displays. Consequently, such threedimensional arrangements can also instrumentalize the highly-developed skills of human spatial gestalt perception ${ }^{13}$ for navigation and reasoning. Technically speaking, macro-architectures connect different complex InfoVis tableaus like a three-dimensional concept map, using different visualization scenarios or layouts as nodes, and connecting them to various conceptual relations.

When implemented in a visual and conceptually consistent way, the creation of such a visual system scaffold can operate like an advance organizer ${ }^{14}$ to facilitate the overall cognitive orientation and navigation between different views, mediate macro- and micro-perspectives as

13 Stemming from the German word "Gestalt", meaning form, the theory of gestalt perception describes, how our visual perceptual system identifies figures from the organization of visual cues.

14 An advance organizer is a pre-sketch of some material in advance to the detailed processing and allows to integrate all following information into the mental model constructed from the sketch. 
zooming operations, and thus foster the understanding of bigger systems (Schreder et al., 2016). Like for local views, such an architecture can activate natural 3D gestalt perception, perspective taking and reasoning, and can also help to instrumentalize these abilities for abstract reasoning on a macro-level.

In the following section, we outline a conceptual draft on how to generate a visuo-spatial metalayout for complex government systems. Building on this conceptual scaffold, we will discuss how further linking techniques could be interwoven, to merge into a multi-methodically linked compound visualization.

\section{Drawing Things Together: Re-Linking Citizens and Collectives}

By the means of various InfoVis methods (sections 2.1 to 2.7), governmental data constellations can be transformed into locally well-defined graphic representations and layouts tat support visual model-building and model-based reasoning. By the means of various linking techniques (sections 3.1 to 3.4), local data models can be interconnected to achieve representational synergies on a higher level of complexity. In the following, we outline how a visuo-spatial macro-architecture could emerge from linking system elements and local layouts into a spatially integrated threedimensional graph.

Figure 14 draws together multiple methods seen above, including set diagrams (2.1), concept maps (2.2), force-directed graphs (2.3), charts (2.4), and maps - and combines and relinks them, similar to a three-dimensional concept map. To do so - the visual vocabularies of different views (marks, icons, labels, other visual variables) must be aligned and coordinated. Starting from a setdiagram as base layout (main view, top right), government architectures could be visualized as cybernetic circles above these abstracted territories, with their governance cycles modifying and transforming the common functional network of a collective. Such a simple meta-architecture could subsequently serve as carrier structure for interweaving further linking techniques. Even if such combinations will always have to be tailored to an InfoVis system, the exploitation of multiple linking techniques seems meaningful. Possible methods include:

- Narrative introductions of basic elements (cf. Fig. 2) and visual structures for an incremental construction of an overall mental model of a political system.

- Traceable transitions from data (attributes and relations) into the alternative spatial layouts and visual variables InfoVis diagrams (cf. Figure 10).

- Linking diagrams as artificial arrangements (=InfoVis) with physical arrangements (SciVis) (e.g., sets and maps), to offer a mutual transfer of insights (cf. Sedlmair et al., 2009).

- Visual Storytelling might make use of small multiples (Figure 14, bottom), seamless camera pans or tracking shots for narrative-sequential walkthroughs.

- Hyperlinks to other related representations or media outside the government visualization interface can link the focused system with environmental data and provide InfoVis context (Figure 14, left hand side). 
- Ego-views can offer relatable positions and meaningful entry points into complex information architectures - and facilitate personal access to deeper exploration (cf. citizen pictograms in the center of the spatial macro-architecture).

Personal modification and annotation can furthermore support the individual connection of the presented InfoVis model with existing mental representations - and interweave the external and internal knowledge graphs.

We consider such possible combinations of linking techniques to open new ways and means, to design InfoVis interfaces that are able to deal with complex data across multiple domains, and to more effectively support a coherent mental (macro)model construction on the user's side.

Figure 14: Visuo-Spatial Macro-Architecture for the Integration of Multiple Views on Government Data, bringing multiple InfoVis methods (set diagrams, networks, (concept) maps, statistical charts) and linking techniques (macro-architecture, multiple coordinated views and narration) together.

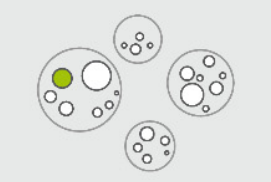

set diagram view $\mathbf{4}$
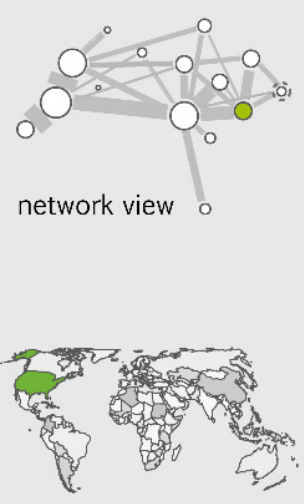

map view

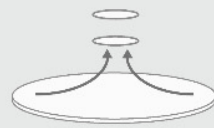

$\longrightarrow$ taxation
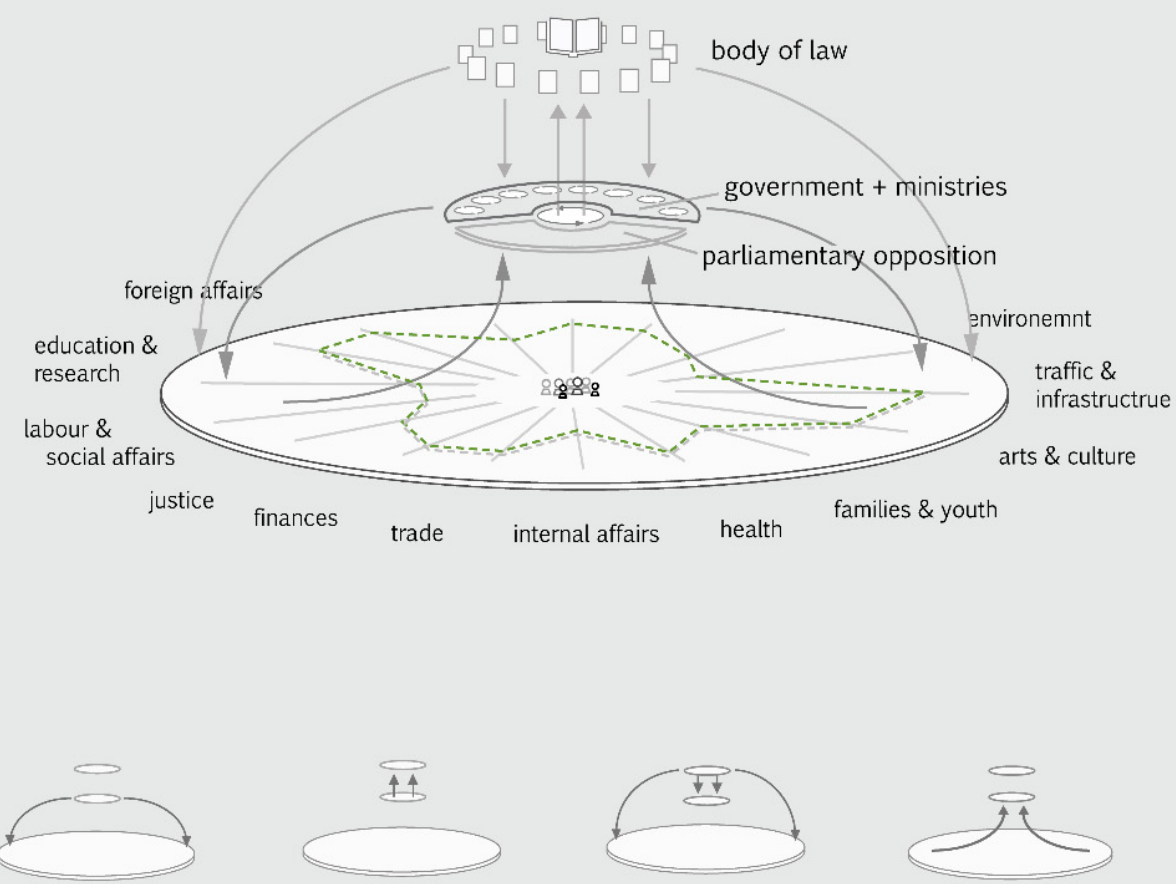

governance

legislation

jurisdiction 
Whether these techniques will help to (cognitively or motivationally) reconnect citizens to their collective self-governing structures and dynamics (i.e., local and global polity, policy, and politics), will become known only by future implementations and evaluations. ${ }^{15}$ At least one can make the argument that the political data, discourse and knowledge domain is among the socially most relevant communication areas to aim for such reconnections. And that it - due to its controversial and polarized character - surely deserves the best reconnection techniques that the InfoVis community has at its future disposal.

\section{Outlook and Discussion}

In this article, we discussed methods on how to ease access to (linked) government data by linking information visualization techniques. While InfoVis libraries offer a variety of well-established methods to visually analyze well-delimited data sets, we see a specific need to develop and promote novel linking techniques to meet the demands of more complex topic constellations, which frequently connect different data dimensions and domains. From a semiotic perspective, this amounts to the development of a higher-level visual syntax. From a Visual Analytics perspective, the endeavor equals the development of Visual Synthetics techniques, to build up the mental macromodels, which again are guiding and facilitating local sense making as top-downstructures for (macro-)model-based reasoning. For the government data and knowledge realm, these coherence techniques can condense into interfaces which support cognitive information integration for linked data by a more consistent macro-representational design. From the application perspective of those professions which we consider as main users of visual communication methods (i.e., civic education, journalism, or OGD initiatives) we expect

- enhanced visual macro-cognition support for teaching and learning - in terms of a more coherent and consistent construction of global mental models),

- a better integrated and contextualized reporting of news - as elaboration, extension and interlinking operations of mental models on political topics and data) and

- a better integration of insights resulting from visual LOGD exploration - in terms of visually supported navigating and reasoning across data domains and cognitive frames.

We demonstrated four different methods on how to connect local layouts into bigger pictures: hyperlinks, narrative guidance, coordinated multiple views, and visual meta-architectures. These techniques serve as linking methods for more interoperable system and data elements - and provide novel means for managing, taming, and communicating LOGD complexity.

15 Regarding the current state of empirical evaluation results, only first fragments are available: Braseveneau and colleagues (2017, in press) recently evaluated a linked open data system with coordinated linkes views. Their participants valued the possibility to generate linked graphics, yet the evaluation design did not allow any causal inferences for linked views, as the tasks did not require reasoning across views. Furthermore, most visualizations of linked data are targeted at experts or tech users, hardly any are designed with lay users - like citizens - in mind (Dadzie \& Pietriga, 2017). 
Abstract discourse and policy controversies immerse non-expert citizens in a cognitively challenging and emotionally charged semiotic environment, to which most of them cannot easily connect. We consider the outlined layout and linking techniques to open new ways to access this complex knowledge domain by offering well-integrated architectures for conducting introductions or elaborations. By the means of such a scaffold, classical policy debates and ongoing controversies can be visually reframed and made newly accessible. This might also foster perspective-taking from different standpoints and thus a mediation between different political opinions and positions. In face of a polarized and partisan discourse and media landscape, we consider such possible future endeavors to be of renewed relevance.

Looking up from the functional field of politics, we finally consider these or similar macromodelling endeavors to be of relevance for any other field of the social sciences, also dealing with heterogeneous phenomena and datasets. From a functionalist perspective, this outline of how to synthesize local views on bigger political systems could serve as an exploratory blueprint that could be transferred and evaluated by similar elaborations for how to make complex systems in other functional subfields more transparent and accessible. As such we hope to see learning cycles between macrocognition-supporting interface developments in different application areas - which could blaze new trails into more complex data visualization landscapes, while keeping cognitive overstretch at bay.

\section{References}

Alani, H., Dupplaw, D., Sheridan, J., O'Hara, K., Darlington, J., Shadbolt, N., \& Tullo, C. (2007). Unlocking the potential of public sector information with semantic web technology. In The semantic web (pp. 708721). Springer Berlin Heidelberg.

Alsallakh, B., Micallef, L., Aigner, W., Hauser, H., Miksch, S., \& Rodgers, P. (2014). Visualizing sets and settyped data: State-of-the-art and future challenges. In Eurographics conference on Visualization (EuroVis)-State of The Art Reports (pp. 1-21).

Ayres, P., \& Sweller, J. (2005). The split-attention principle in multimedia learning. In R. E. Mayer (Ed.), Handbook of multimedia learning (pp. 135-146). New York: Cambridge University Press.

Baeza-Yates, R., \& Ribeiro-Neto, B. (2010). Modern Information Retrieval (2ed edition.). New York: Addison Wesley.

Bikakis, N., \& Sellis, T. (2016). Exploration and visualization in the Web of Big Linked Data: A survey of the state of the art. arXiv preprint arXiv:1601.08059.

Bizer, C., Heath, T., \& Berners-Lee, T. (2009). Linked data-the story so far. Semantic Services, Interoperability and Web Applications: Emerging Concepts, 205-227.

Brasoveanu, A.M.P., Sabou, M., Scharl, A., Hubmann-Haidvogel, A. and Fischl, D. (2017). “Visualizing Statistical Linked Knowledge Sources for Decision Support", Semantic Web Journal, (8)1. 
Card, S., Mackinlay, J., \& Shneiderman, B. (1999). Readings in information visualization: Using vision to think. San Francisco: Morgan Kaufmann.

Chun, S. A., Shulman, S., Sandoval, R., \& Hovy, E. (2010). Government 2.0: Making connections between citizens, data and government. Information Polity, 15(1), 1.

Collins, C. M., \& Carpendale, S. (2007). VisLink: Revealing relationships amongst visualizations. Visualization and Computer Graphics, IEEE Transactions on, 13(6), 1192-1199.

Dadzie, A. S., \& Rowe, M. (2011). Approaches to visualising linked data: A survey. Semantic Web, 2(2), 89124.

Ding, L., DiFranzo, D., Graves, A., Michaelis, J., Li, X., McGuinness, D. L., \& Hendler, J. (2010, March). Datagov Wiki: Towards Linking Government Data. In AAAI Spring Symposium: Linked data meets artificial intelligence (Vol. 10).

Dorling, D. (2011). Area cartograms: their use and creation. In M. Dodge, R. Kitchin, C. Perkins (Eds.) The Map Reader: Theories of Mapping Practice and Cartographic Representation (pp. 252-260). Hoboken, NJ: Wiley-Blackwell.

Dörk, M., Feng, P., Collins, C., \& Carpendale, S. (2013). Critical InfoVis: Exploring the politics of visualization. In Proceedings of CHI'13 Human Factors in Computing Systems (pp. 2189-2198).

Engelhardt, Y. (2006, June). Objects and spaces: The visual language of graphics. In International Conference on Theory and Application of Diagrams(pp. 104-108). Springer Berlin Heidelberg.

Few, S. (2004). Show me the numbers: Designing Tables and Graphs to Enlighten. Oakland, CA: Analytics Press.

Finer, S. E. (1997). The History of Government (Vol I - III). Cambridge University Press.

Gantz, J. \& Reinsel, D. (2011). Extracting value from chaos. IDC iview 1142: 1-12.

Harman, G. (2014). Bruno Latour: Reassembling the Political. Pluto Press.

Heer, J., Bostock, M., \& Ogievetsky, V. (2010). A tour through the visualization zoo. Commun. ACM, 53(6), 59-67.

Janssen, M., Charalabidis, Y., \& Zuiderwijk, A. (2012). Benefits, adoption barriers and myths of open data and open government. Information Systems Management, 29(4), 258-268.

Johnson-Laird, P. N. (1980). Mental models in cognitive science. Cognitive science, 4(1), 71-115.

Kitchin, R., \& Freundschuh, S. (2000). Cognitive mapping: Past, present, and future (Vol. 4). Psychology Press.

Klein, G. \& Hoffman, R. R. (2008) Macrocognition, mental models, and cognitive task analysis methodology. In Schraagen (ed.) Naturalistic Decision-making and Macrocognition, pp. 57-80.

Kobsa, A. (2004). User experiments with tree visualization systems. In Information Visualization (InfoVis) 2004. IEEE Symposium on (pp. 9-16).. 
Klyne, G., \& Carroll, J. J. (2006). Resource description framework (RDF): Concepts and abstract syntax.

Krempel, L., \& Plümper, T. (2003). Exploring the dynamics of international trade by combining the comparative advantages of multivariate statistics and network visualizations. Journal of Social Structure, 4(1), 1-22.

Liu, Z., \& Stasko, J. T. (2010). Mental models, visual reasoning and interaction in information visualization: A top-down perspective. IEEE Transactions on Visualization \& Comput. Graph., 16, 999-1008.

Ngomo, A. C. N., \& Auer, S. (2011). Limes-a time-efficient approach for large-scale link discovery on the web of data. integration, 15,3 .

Peña, O., Aguilera, U., \& López-de Ipina, D. (2015). Linked open data visualization revisited: a survey. Under Review at Semant. Web J.

Ragan, E. D., Endert, A., Sanyal, J., \& Chen, J. (2016). Characterizing provenance in visualization and data analysis: an organizational framework of provenance types and purposes. IEEE transactions on visualization and computer graphics, 22(1), 31-40.

Ram, S., \& Liu, J. (2009). A new perspective on semantics of data provenance. In Proceedings of the First International Conference on Semantic Web in Provenance Management-Volume 526 (pp. 35-40). CEURWS. org.

Roberts, J. C. (2007). State of the art: Coordinated \& multiple views in exploratory visualization. In Coordinated and Multiple Views in Exploratory Visualization, 2007. CMV'07. Fifth International Conference on (pp. 61-71). Washington, DC: IEEE Computer Society Press.

Rose-Ackerman, S. (2013). Corruption: A study in political economy. Academic Press.

Schreder, G., Windhager, F., Smuc, M., Mayr, E. (2016). Supporting Cognition in the Face of Political Data and Discourse: A Mental Models Perspective on Designing Information Visualization Systems. In Proceedings of the Sixth International Conference for eDemocracy and Open Government (CeDEM 2016): pp. 213-218, IEEE Computer Society, Los Alamitos, CA.

Schmachtenberg, M., Bizer, C., Jentzsch, A., \& Cyganiak, R. (2014). Linking Open Data cloud diagram, Retrieved from: http://lod-cloud.net/

Sedlmair, M., Ruhland, K., Hennecke, F., Butz, A., Bioletti, S., \& O'Sullivan, C. (2009). Towards the big picture: Enriching $3 \mathrm{~d}$ models with information visualisation and vice versa. In International Symposium on Smart Graphics (pp. 27-39). Springer Berlin Heidelberg.

Segel, E., \& Heer, J. (2010). Narrative visualization: Telling stories with data. Visualization and Computer Graphics, IEEE Transactions on, 16(6), 1139-1148.

Shneiderman, B. (1996). The eyes have it: A task by data type taxonomy for information visualizations. In Visual Languages, 1996. Proceedings of IEEE Symposium on (pp. 336-343). IEEE.

Stichweh, R. (2013). The history and systematics of functional differentiation in sociology. Bringing Sociology to International Relations. World Politics as Differentiation Theory, 50-70. 
Tversky, B. 1993. Cognitive maps, cognitive collages, and spatial mental models. In Spatial Information Theory. A Theoretical Basis for GIS, A. U. Frank \& I. Campari, Eds. Springer, Berlin. 14-24.

Ubaldi, B. (2013). Open Government Data: Towards Empirical Analysis of Open Government Data Initiatives, OECD Working Papers on Public Governance, No. 22, OECD Publishing.

Valsecchi, F. Abrate, M., Bacciu, C., Tesconi, M. \& Marchetti, A. (2015) Linked Data maps: Providing a visual entry point for the exploration of datasets. In Dhavalkumar Thakker, Daniel Schwabe, Kouji Kozaki, Roberto Garcia, Marco Brambilla, and Vania Dimitrova, (eds.), IESD 2015: Proceedings of the 4th International Workshop on Intelligent Exploration of Semantic Data, 2015.

Villazón-Terrazas, B., Vilches-Blázquez, L. M., Corcho, O., \& Gómez-Pérez, A. (2011). Methodological guidelines for publishing government linked data. In Linking government data (pp. 27-49). Springer, New York.

Windhager, F. (2013). On Polycubism. Outlining a dynamic information visualization framework for the humanities and social sciences. In M. Füllsack (Ed.). Networking Networks. Origins, Applications, Experiments (pp. 28-63). Vienna, Austria: Turia + Kant.

Windhager, F. \& Smuc, M. (2014) The Arts of the Possible. Information Visualization in the Field of Politics. Journal of e-Democracy and Open Government 6(2), 151-165.

Windhager, F.; Schreder, G.; Smuc, M.; Mayr, E. (2015) Drawing Things Together: Supporting Information Visualizations' Coherence across Multiple Views. In Proceedings of the IEEE Information Visualization Conference 2016 (Posters Compendium), IEEE Computer Society Press

\section{About the Authors}

\section{Florian Windhager}

Florian Windhager works as a research associate at the Department for Knowledge and Communication Management at Danube University Krems, Austria. He obtained his degree in Philosophy (M.A.) from the University of Vienna. His research interests include applications and cognitive foundations of information visualization, visualization in the digital humanities, and concept development for visual analytics interfaces.

\section{Eva Mayr}

Eva Mayr graduated in Psychology from the University of Vienna in 2004, and completed her doctorate in Applied Cognitive and Media Psychology at the University of Tuebingen, Germany, in 2009. From 2002 to 2005, she worked at the Institute of Psychology in Vienna. Since 2008, she has been a research associate in the Department of Knowledge and Communication Management at the Danube University Krems, Austria. Her research interests focus on how new media technologies can support cognitive processing and informal learning.

\section{Günther Schreder}

Günther Schreder works as a research associate at the Center for Cognition, Information and Management, Danube University Krems, Austria. He received his diploma in psychology at the University of Vienna, Austria. His research and teaching activities focus on user-centered design with respect to non-expert 
users, narrative information design and the development and investigation of cognitive models of narrative communication and organizational culture.

Michael Smuc

Michael Smuc graduated in Psychology at the University of Vienna with an emphasis on methodology, empirical research and cognitive science. He worked for several years as a traffic psychologist with the Austrian Road Safety Board, managing interdisciplinary projects addressing driver interaction with new technologies and Telematics. In 2007, he joined the Danube-University Krems, Austria as a research associate focusing on usability, human computer interaction and participatory information design. 